\title{
MODELING THE STRUCTURE OF COLLABORATIVE NETWORKS: SOME CONTRIBUTIONS
}

\author{
António Lucas Soares ${ }^{1,2}$, Jorge Pinho de Sousa ${ }^{1,2}$, Francisco Barbedo ${ }^{3}$ \\ INESC Porto ${ }^{1}$, Faculty of Engineering, University of Porto ${ }^{2}$, Arquivo Distrital do Porto ${ }^{3}$,
}

PORTUGAL

Collaborative Networks $(C N)$ and Virtual Organisations (VO) can partially be modelled by using Graph Theory, as a natural framework for describing relationships, interactions and collaboration. Our work aims at extending and integrating results from that theory and other different fields and scientific disciplines, to enable modelling complex and non tangible factors, dynamics, trade-offs between individual and common objectives, arising in the set-up and management of VO. As a way to handle these issues in a holistic way, we present extensions of Social Actors Networks that are able to encompass various, complementary perspectives in dealing with VO, and coping with the different phases of its life cycle. Current results seem promising in the direction of creating a sound framework for modelling and supporting holistic process management in Collaborative Networks. For illustration purposes, a simple case study is briefly sketched and some findings presented. Finally some open issues are surveyed and lines for future research identified.

\section{INTRODUCTION}

Networks as a dominant organising principle have always been a subject of enormous interdisciplinary research. In business there is a variety of networks, reflecting different types of collaboration and relationships. Recently the "smallworld" principles have "shown" that seemingly distant, disconnected, and disparate populations, events or actions, can be easily linked to one another (Barabási, 2002).

In organisational terms, there is nowadays an increased interest in looking for "hidden networks" within complex systems, and in supporting organisations to manage their networks as a natural way to create value. The concept of Virtual Organisation (VO) requires new tools and methodologies to support individuals and institutions to create just-in-time activity and exploit opportunities.

Most of the research work on the broad domain that encompasses "Supply Chain Management", "Virtual Organisations", "Collaborative Networks" or related topics, has basically been concentrated on issues such as distributed information technology infra-structures and distributed information systems architectures, novel forms of organisational structures facilitated by those technological advances, new business processes based on inter-organisational relations, etc.

However, much less attention has been paid to important issues related to interorganisational co-operation, and to the particular roles of the actors (individuals and groups). Nevertheless, relationships between enterprises will continue to involve 
people in key managing activities, taking operational, tactical and strategic decisions supported by conveniently distributed information systems.

In fact, collaborative networks are rich structures where the plural facets of actors should be considered, not only to exploit the opportunities of a specific business relationship, but also in order to construct more durable and rewarding links.

This view asks for a broader, multi-perspective approach that takes into account several aspects of a business relationship, some of them not easy to quantify. These should involve issues that range from type and quantity of shared information, commercial and personal trust, subjective assessment of business transactions, etc.

This work aims at contributing for the design of a conceptual framework to enable different entities (small companies, individuals, ...) to establish a certain number of multi-attribute relationships that can be dynamically evaluated and updated, and that will be used as a basis for setting up "formal" and "informal" agreements in the process of managing collaborative networks and virtual organisations.

\section{COLLABORATIVE BUSINESS}

Collaborative Networks $(\mathrm{CN})$ are becoming one of the most powerful strategic business trends. Major changes in the economy are taking place, involving a move from vertically integrated companies towards flexible network organizations, and the ability to quickly and efficiently set-up, maintain, develop and dissolve partnerships with business partners - networkability - is a critical success factor (Österle et al., 2000). In fact, enterprises of all sizes are collaborating more than ever to meet the demands of their customers. Collaboration spanning customers, suppliers and business partners is crucial for developing the right products, for the right markets, at the right time.

The need to go beyond traditional internal business processes, and tackle larger and more complex cross-enterprise processes, has emerged as a natural way to increase flexibility and efficiency. In this new business strategy, that can broadly be called "collaborative business" (c-business), business partners in the whole value network collaborate, sharing information and processes, to provide a higher value product/service, with increased profitability.

C-business is a key enabler to make this happen (Gartner Group, 2001) and it is based on the enhanced connectedness of participants in Internet-based value networks, and, as such, may be regarded as an evolution of e-business. Naturally, cbusiness leverages the potential for value creation in e-business by enhancing opportunities for efficiency gains, exploitation of complementarities, customer and partner lock-in, and the introduction of novel products, services, processes and new business and revenue models (Amit and Zott, 2001).

Collaboration became very popular as a label for a wide variety of organisational forms, the term being therefore extremely vague. An encompassing definition of collaboration is proposed in (Hall, 1999): "Collaboration is broadly defined as the interaction among two or more individuals and can encompass a variety of behaviours, including communication, information sharing, coordination, cooperation, problem solving, and negotiation". 
On the purely technological side, one big challenge is clearly the "integration" of collaboration services with other technologies, in particular ERP, in order to offer solutions that simultaneously and coherently support business and work processes. This should be achieved along with integration and optimisation of internal and external business processes, somehow leading to a new generation of ERP solutions, the so-called ERP II systems. But on the business side, the implications are that new forms of understanding, modelling and formalising collaboration are needed, so that practical complex requirements are taken into account.

When we deal with physically distributed business processes, the way partners interact becomes critical, as they do not necessarily share the same applications, integration technology or even operation or management concepts. Moreover external control over inside applications will in general be excluded. The collaborating processes must therefore be explicitly represented so that multiple businesses can support their execution on multiple systems (CSC 2002), i.e. process management should be a homogeneous capability in a heterogeneous environment.

Business process modelling languages allow the description and execution of business processes and support the design of software systems. The Business Process Management Initiative (BPMI.org) is probably the most relevant proposal in the domain). However, in order to reach higher levels of business integration, a lot has still to be agreed on process representation formalisms. The contributions of this work towards more general, holistic representations of collaborative business processes follow this trend.

\section{GRAPH THEORY}

Graph Theory has an enormous potential in modelling Collaborative Networks (CN) and Virtual Organizations (VO), as a natural framework for representing relationships, interactions and collaboration. This framework is supported by a graphical representation that enhances the understanding and sharing of relationships, supporting network management methods and algorithms. Graphs are natural models for all types of networks (Ahuja et al., 1993) and are based on an old, well-understood graphical metaphor, based on nodes and edges (or arcs).

Nodes usually represent entities or objects (companies, departments, individuals, or other types of "actors"). Edges represent relationships, interactions, flows (physical, information, cash, ...). One may assign all sorts of attributes (numerical, textual) to the entities or nodes of the graph, in order to model competences (skills or "capabilities"), "values" or importance, physical location, "trust level" or "history". Similarly, edges may be assigned distances, capacities, costs, logistic means, ...

Then, by using traditional algorithms, more complex measures such as the "connection capital", "centrality", "groupability", can be calculated from the graph, if appropriate values are assigned to the connections. Both nodes and edges may have several attributes that may for example quantify the average amount of exchanged information, the level of trust between partners, or the degree of ownership between companies. This "open" view of a network should allow us to model quite different situations and problems, such as client/supplier or co-operative 
planning environments, technical co-operation, informal information exchange, interest groups, etc.

There are a lot of commercially available network optimisation tools; packages for supporting network design; and software for drawing several types of networks. These tools are used for modelling interactions and quantitative relationships, but they have in general a very limited capacity to represent non tangible, qualitative relationships (such as the level of trust or the exchange of tacit information); and to deal with multiple criteria in evaluating different operating alternatives.

The basic operations on graphs are associated to: flows (based on capacities on the edges); paths (based on distances, costs, ...), related to problems of minimising distances, identifying the shortest paths, minimising costs; connections, defining problems such as finding paths linking entities with given properties, or defining clusters. In this latest group some classical GT models may be quite useful in understanding the structure and the operation of $\mathrm{CN}$ and VO. E.g. a spanning tree in a given network is a connected subgraph (i.e. a subset of the original edges) that "spans" all nodes, and has no cycles. This idea may be useful in trying to find ways of linking together the entities, through a "linking infrastructure" of minimum cost, or in understanding how information propagates along a network.

\section{SOCIAL ACTORS NETWORKS}

The concept of Social Actors Networks (SAN) (Lemieux, 1999) provides us with a complementary tool for modelling VO. It may be considered as a good way for understanding which organisational elements (social actors) are involved, how these elements are related to each other, and what kind of relationships exist between them. It should also help us in characterising social actors, and their relationships.

Social actors are individuals or groups capable of performing activities and of interacting with other actors in order to pursue goals. They are represented as the nodes of the network and the relationships are represented as arcs or edges.

SAN can be viewed as an approach to Business Network Modelling, focusing on the analysis of the social and organizational structure of collaborative networks. Examples of what can be analysed are: structures of interaction (logistics, informational, etc.); elements of sociability (connectiveness, trust, awareness, information paths, etc.); power structures (who controls who).

\subsection{Organizational analysis perspectives}

SAN can be particularly useful in organizational (re)design, and reports of its effective use in that area are not new. For example, Tichy (1981) evaluates organizations using several network strategies after identifying groups ("coalitions" and "cliques") as well as their connections. It is then possible through SAN based methods to determine which groups are dominant, how they agree or disagree and, consequently if it is required to adopt cooperation strategies or conversely it is more adequate to establish negotiation scenarios. In general terms it is possible to use SAN to analyse organizational views, e.g. social, political, cultural, technological, at different levels - inter-organizational, organizational, group, individual (Tichy, 1981), and to help us in revealing the "informal" organizational chart (Mintzberg and Heyden, 1999). 


\subsection{SAN analysis in the context of inter-organizational processes}

Different analysis levels are possible with SAN (see e.g. Soares and Sousa, 2002). SAN can be used to support the modelling of $\mathrm{VO}$ or $\mathrm{CN}$ focusing on their structural aspects: which organisational elements (social actors) are involved; how these elements are related to each other; what kind of relationships exist between elements. For example, in a supply chain, all the participating companies must be known, as well as their client-supplier relations. They should also provide for example an identification of companies with the same supplying characteristics (types of products, delivery performance, supply chain tier, ...). SAN can also focus on the relational aspects to characterise social actors and the relationships between them. This might involve for example the evaluation of logistic issues between two supply chain commercial partners (such as delivery lead times, quality performance), of the degree of trust between partners or of the degree of information exchange concerning several subjects. At the individual level, SAN might be used to evaluate the importance of an actor (e.g. measured by the centrality of his position concerning information exchange in the network) or the potential for an actor to establish relationships in the network.

\subsection{Combining social structure with process analysis}

The role of a SAN in the analysis and design of inter-organizational processes is to reveal how the structure of the social relations between the actors performing the activities in the process can influence either positively or negatively the performance of the process. Here one crucial aspect is the identification and characterisation of the social actors. The approach followed in this work was to select the social actors based on the participation and/or influence in the inter-organizational processes. This implies that the first step in data collection is the processes identification, which is a complex task on its own (see e.g. Eriksson and Penker, 2000).

\section{CASE STUDY}

This section describes the application of the concepts and methods briefly described above in the analysis and design of a record-keeping network, in the specific sector of Port Wine commercialization, in Portugal. The main goal of the study was to analyse a network of record-keeping organizations in order to reengineer their processes. This would involve, besides the business processes specification, the analysis and design of an informatics infrastructure for the network. It was then decided to depart from the following research questions:

Can a SAN based method be effective in the analysis of a social structure of a $\mathrm{CN}$ in the record-keeping sector? Can a SAN based method be combined with a business architecture modelling method to create a more comprehensive approach to inter-organizational business processes (re)design?

These two questions were the starting point of a spiral cycle of plan $>$ act $>$ observe $>$ reflect aimed at solving the network problems from one side, and to create theoretical and practical knowledge from the other side.

\subsection{Organizational, social and legal context}

Three organizations compose the network: ADP, the record-keeping of Porto, IVP, the Port Wine Institute and EVP, a company commercialising Port Wine. IVP and 
EVP business is exclusively related with the port wine product, while ADP business is information and culture. EVP is private; the other two are public organizations. IVP and EVP are inherently linked by a legal framework, meaning that most of the social and functional relationships are imposed by a superior authority. This constrains the types of social relationships as well as the types of interorganizational processes to be performed. Nevertheless, the way in which the processes are executed is prone to some variability. It is on this issue that the SAN analysis will bring some input regarding the optimisation of those processes.

\subsection{Actors selection and aggregation level}

Actors have been included in the network either because they were participating in an inter-organizational process or because they could by some means influence its execution. The selected level for the aggregation of actors was the functional unit. This is a compromise between departmental units which would be too coarse for the required analysis and the individual role that would cause an explosion of actors and would add unnecessary complexity to the models. The methods used to collect data were semi-structured interviews, observation, document analysis and group discussions. The last method was fundamental to involve the relevant people in the analysis process aiming at incorporating a more interpretative dimension to the analysis.

\subsection{SAN analysis}

Based on reported experiences (Ajuha and Carley, 1998; Hagen et al., 1998), a set of measures for the analysis was selected as follows:

General measures for characterisation of the network as a whole - cohesion, density, transitivity;

Individual measures that take as a referential an individual actor and the connecting categories with the rest of the set - adjacency, geodesic, connectivity and maximum flow;

Centrality measures were used to determine the core actors in the network, the ones crucial for the development and support of the inter-organizational network;

Subgroup identification measures for a nClique analysis.

The global analysis enabled the identification of the actors with higher weight and actors that are relatively isolated. The network has a unique component (it is a completely connected sub-graph).

The individual analysis provided results on adjacency, number of geodesics, maximum flow and connectivity, centrality, proximity and intermediation. The most central actor in the network was identified. This confirms the empirical and perceptual data and it is very simply explained by the relevance of supervisory and control activities.

In the subgroup analysis (nCliques), the goal is to identify sets of actors that have a high cohesion, that act in a reciprocal way, that share the same social values. This measure points to actors that are closely aggregated through the execution of functions or tasks, or through informal relationships, even if included in processes or institutional activities. From the inclusion of certain actors in a clique we may infer the existence of a degree of cohesion based both on the specialization of tasks and in the co-participation in inter-organizational processes. For example, some cliques included actors clearly specialized in technological activities, side by side with 
actors playing roles of coordination and administrative support to the core set of specialized tasks.

Two general conclusions should be highlighted. First, there is a discrepancy between the actors identified as more influent and central in the network and those that have more power as concluded through the centrality of intermediation measure. This can be explained by the fact that centrality of hierarchy within a network is better analysed by the influencing capacity than by the degree of active participation.

Secondly, the high centrality and weight of an actor corresponds to a frequent participation in inter-organizational processes. This means that the information infra-structure should reflect this fact. Similarly, the distribution pattern of cliques by specialization areas can be a starting point for the definition of the information system supporting the inter-organizational processes, e.g. workflow patterns.

In the practical case study, the analysis of the network enabled the identification of a multiplex relationship between two particular actors, encompassing connections where control is exerted unilaterally upon one actor, but also transactional interactions where resources are exchanged across the connection. Centrality measures show those two actors occupy central positions, showing therefore they play an important role in the network. From the process models (forming an additional analysis dimension), a set of improvements was proposed that were considered technologically and organizationally feasible. The next step, enabled by the SAN results, was to analyse the constraints for the implementation of the improvements required by the "social structure".

Summarising, it was concluded that a substantial change was needed in the relationships between those two entities namely to reduce the intensity of control, and to allow the implementation of a computer based system to improve the process under consideration.

\section{CONCLUSIONS AND FURTHER WORK}

Graph Theory and Social Actors Networks seem to be able to capture key features of the "networked reality", and consider multiple perspectives and criteria. Moreover the resulting models are easy to understand and "share", are flexible and easy to customise, providing quantitative results that can support the set-up and management of VO. Measures such as the "connection capital", "centrality", "groupability", can be calculated from the graph, if appropriate values are assigned to the connections. These measures will help to characterise in more detail the social actors and their relationships.

Further research is needed to tackle more complex logistic issues and "true" multi-criteria, multi-perspective decision-making environments. More general (adaptive) algorithms should be designed as part of broader Decision Support Systems for tackling complex planning processes of large supply chains and collaborative networks. The approaches traditionally used in the field of Multiple Criteria Decision Making, by explicitly dealing with multiple perspectives, may therefore give interesting contributions to the models of Graph Theory. Moreover in order to better capture non tangible and qualitative aspects of reality, Fuzzy Set Theory and Fuzzy Logic could probably be, at least partially, incorporated into the above approaches, thus creating more powerful analysis and design tools. Another 
important issue insufficiently addressed so far is the inclusion of information technology and systems in the social structure and process models. An outline of a possible approach was already pointed out by Soares $e$ t al. (2001) for systems based on software agents architectures. In this particular case, a new and challenging requirements analysis and specification approach will be possible. Some interaction features and other attributes of a community (or society) of software agents can also be modelled by SAN, leading to a coherent and uniform analysis method, opening some new and challenging analysis possibilities.

Acknowledgments. The work presented in this paper was partially developed within the European Project THINKcreative - Thinking network of experts on emerging smart organizations (IST-2000-29478). The authors would like to thank the European Commission for funding, and their project partners for suggestions and comments.

\section{REFERENCES}

Ahuja, R., Magnanti, T., Orlin, J., 1993. Network Flows - Theory, Algorithms and Applications, Prentice-Hall Inc.

Ahuja, K. M., Carley, M. K., 1998. "Network Structure in Virtual Organizations", Journal of Computer Mediated Communication, 3(4). In http://www.ascusc.org/jcmc/

Amit, R., Zott, C., 2001. "Value Creation in e-Business", Strategic Management Journal, vol. 22, pp. 493-520.

Barabási, A.-L., 2002. Linked: The New Science of Networks, Perseus, Publishing, 2002,

2.parag CONTEXT Mark Buchanan, Small Worlds and the Groundbreaking Science of Networks, W.W. Norton \& Company.

Eriksson, Penker, 2000. UML Business Modelling: Business Patterns. Wiley.

CSC 2002. "The Emergence of Business Process Management, a report by CSC's Research Services" - version 1.0.

Gartner Group, 2001. "Attention SMBs: Plan for Collaborative Commerce Now". Top View.

Hagen, G., Killinger, D. K., Streeter, R. B., 1997. “An Analysis of Communication Networks Among Tampa Bay Economic Development Organizations". Connections 20(2) In http://www.analytictech.com/connections/v20(2)/cover.htm.

Hall, T., 1999. “Intelligence Community Collaboration, Baseline Study Report". In http://collaboration.mitre.org/prail/IC_Collaboration_Baseline_Study_Final_Report/toc.

Lemieux, V., 1999. Les réseaux d'acteurs sociaux, Presses Universitaires de France.

Mintzberg, H., Heyden, L. van der, 1999. "Organigraphs: Drawing how Companies Really Work". Harvard Business Review.

Österle, H., Fleisch, E., Alt, R. 2000. Business Networking: Shaping Enterprise Relationships on the Internet. Springer.

Soares, A. L., Toscano, C., Sousa, J. P., 2001. "A Social Actors Network Approach for the Design of Networked and Virtual Enterprises". In Kovacs, Bertok and Haiddeger (Editors) Digital Enterprise Challenges: Life-Cycle Approach to Management and Production, Kluwer.

Soares, A. L., Sousa, J. P., 2002. "Multiple Perspective Configuration of Virtual Enterprises Using Social Actors Networks". In Camarinha-Matos L. M. (Editor) Collaborative Business Ecosystems and Virtual Enterprises, Kluwer Academic Publishers.

Tichy, N. M., 1981. "Networks in Organizations". In Nystrom, P., Starbuck, Haynes, W. (Editors), Handbook of Organizational Design. Vol. 2: Remodeling Organizations and their Environments. Oxford University Press. 